https://doi.org/10.1016/j.brainres.2018.06.014 @2018 This manuscript version is made available under a CC-BY-NC-ND 4.0 license http://creativecommons.org/licenses/by-nc-nd/4.0

\title{
Bilateral cortical representation of tactile roughness
}

Genna C. ${ }^{1}$, Oddo C. ${ }^{1}$, Fanciullacci C. ${ }^{1}$, Chisari C. ${ }^{3}$, Micera S. ${ }^{1,2}$, Artoni F $\mathrm{F}^{1,2, *}$

${ }^{1}$ The BioRobotics Institute, Scuola Superiore Sant'Anna, Pisa, Italy

2 Translational Neural Engineering Laboratory, Center for Neuroprosthetics, École Polytechnique

Federale de Lausanne, Switzerland

${ }^{3}$ Neurorehabilitation Unit, University Hospital of Pisa

* Corresponding Author 


\section{Abstract}

Roughness is the most important feature for texture discrimination. Here we investigate how the bilateral cortical representation of touch is modulated by tactile roughness by analyzing the neural responses elicited by stimuli with various coarseness levels ranging from fine to medium.

A prolonged stimulation was delivered to 10 healthy subjects by passively sliding a tactile stimuli under the fingertip while recording the EEG to study the modulation of Somatosensory Evoked Potentials (SEPs) as well as activity in the theta and alpha bands. Elicited long-latency SEPs, namely bilateral P100N140 and frontal P240 were consistent across stimuli. On the contrary, the temporal lag N140 - P240 was nonlinearly modulated both in contralateral and ipsilateral sides, in agreement with literature. Using a time-frequency analysis approach, we identified a theta band power increase in the $\left[\begin{array}{ll}0 & 0.5\end{array}\right] \mathrm{s}$ interval and a partially overlapped power decrease in the alpha band which lasted throughout the stimulation. The estimated time these two phenomena were overlapped was comparable across stimuli, whereas a linear decrease in alpha band amplitude was reported when increasing the stimulus roughness in both contralateral and ipsilateral sides.

This study showed that the selected tactile stimuli generated physiological bilateral responses that were modulated in a diversified way according to the stimulus roughness and side. Specifically, we identified sensory processing features (i.e., theta and alpha time overlap) invariant to the stimulus roughness (i.e., associated to a basic cortical mechanism of touch) and roughness-dependent cortical outputs comparable in the contralateral and ipsilateral sides that confirm a bilateral processing of tactile information.

Keywords: touch, bilateral, somatosensory cortex, roughness, EEG 


\section{Introduction}

Touch enables us to manipulate objects (Johansson and Flanagan, 2009) and recognize their properties such as shape and symmetry (Ballesteros and Reales, 2004; Klatzky et al., 1985).

In the haptic perception of materials, roughness is the most important feature for discrimination of textured surfaces and thus salient to the sense of touch (Bergmann Tiest and Kappers, 2007; Taylor and Lederman, 1975; Weber et al., 2013).

Recently, psychophysical protocols have been combined with neurophysiological studies to provide a better understanding of the afferent peripheral neural mechanisms that produce the spatial and temporal codes mediating tactile perception (Connor et al., 1990; Oddo et al., 2016; Weber et al., 2013; Yoshioka et al., 2001).

Several studies showed that the cortical processing of roughness discrimination is organized hierarchically and follows two principal schemes (Kitada et al., 2005): i) the cognitive-based processing (i.e., roughness estimation task) (Burton et al., 1997) that generates activation in prefrontal area (i.e., active discrimination of surfaces) (Bodegård et al., 2000; Harada et al., 2004; Stoeckel et al., 2003); ii) the sensory processing (i.e., roughness no-estimation task) that involves mostly the somatosensory area (Coghill et al., 1994; Francis et al., 2000; Roland and O'Sullivan, 1998).

A number of studies have examined the global somatosensory response using fMRI (functional Magnetic Resonance Imaging) (Arthurs et al., 2000; Backes et al., 2000; Nelson et al., 2004) and MEG (Magnetoencephalography) (Iguchi et al., 2002; Torquati et al., 2002). In general, they indicate the existence of a relation between the stimulus intensity and the intensity of the evoked signal as well as the activated volume of cortex. 
Electroencephalography (EEG) can provide additional information not only on the amplitude and topography of the brain responses but also on the latency of the evoked potentials (Munoz et al., 2014). Furthermore, the EEG allows monitoring Somatosensory-Evoked Potentials (SEPs), which represent the direct cortical response of the Central Nervous System to sensory stimuli (Allison et al., 1992; Salenius et al., 1997). SEP characterization is also fundamental to identify which components are involved in the roughness discrimination task and which ones reproduce the same features across stimuli (Ballesteros et al., 2009; Munoz et al., 2014).

Given the concurrent activation of several processes, it is currently unclear how to determine what amounts to be a separation between processing related simply to touch from that related to finegrained texture discrimination. The hypothesis behind the study is that, by carefully tuning a specific parameter of tactile stimulation (roughness) in carefully controlled conditions, it is possible to disentangle the role these processes have in the tactile discrimination of surfaces. Understanding these role has important implications in neuroprosthetics, e.g. to assess the cognitive workload required for the control of the prosthesis (Deeny et al., 2014). The identification of the hallmarks of texture discrimination may also provide objective evidence of a successful restoration of homologous tactile feedback via somatotopic intraneural stimulation (Kaczmarek et al., 1991; Oddo et al., 2016).

In our previous study (Genna et al., 2017), we proposed a tactile stimulation consisting in a passive dynamic stimulation that replicates a controlled sliding action of the human fingertip during the recording of EEG activity. This stimulation has two main advantages: i) record the bilateral brain responses generated by the activation of the full range of mechanoreceptors; ii) monitor the dynamics of the brain responses to a prolonged stimulation.

Here we delivered tactile stimuli with different levels of roughness and we studied of the whole brain activation elicited by the different stimuli, both in the time and time-frequency domains. The goal of this study was to evaluate the cortical mechanisms underlying fine-grating tactile stimulation and disentangle 
them from the processes related to the sensory processing of touch. We followed a similar EEG analysis scheme to that in (Genna et al., 2017), and used approaches both in time and frequency domains as follows. In the time domain, the passive sliding of a finger over a surface generated long-latency SEPs consisting in a bilateral P100 - N140 sequence located in the somatosensory area, a pre-frontal P240 and a central peak at 200ms after the end of the stimulation (Genna et al., 2017).

For the time-frequency analysis we computed the somatosensory Event-related Synchronization (ERS) and Desynchronization (ERD) (Pfurtscheller and Lopes da Silva, 1999). In (Genna et al., 2017) we identified two consecutive cortical phenomena modulating two different bands: i) increase of power in the theta band only in the initial stimulation phase (i.e., below 500ms); ii) bilateral decrease of alphaband power throughout the stimulation.

We compared the activation of the contralateral and ipsilateral hemispheres to observe whether tactile roughness modulated SEP amplitude/latency and the related somatosensory ERS/ERD across stimuli.

\section{Results}

\subsection{SEP characterization}

Each stimulus generated long-latency SEPs comparable to our previous study (Genna et al., 2017) (see Fig. 2).

The contralateral ROI (see CLH-ROI in Fig. 2A) clearly identified the temporal sequence P100-N140 across stimuli in the following time interval: [60 120]ms for the P100 and [120 220]ms for N140. The identified SEPs reported slight differences across stimuli in the amplitude of the scalp topographies. Furthermore, for all four stimuli, the scalp topographies confirmed that P100 and N140 SEPs were generated in the contralateral somatosensory cortex (see scalp topographies in Fig. 2A). 
The ipsilateral ROI (see ROI - IL in Fig. 2B) showed also clear and delayed SEP responses, with respect to the contralateral side, for both P100 and N140 (see Fig. 2B). While more oscillations with respect to ROI-CL could be observed, the positive and negative deflections were still clearly detectable. The P100 showed a distinct ipsilateral activation in SP-650 and SP-1050, whereas stimuli SP-450 and SP-800 generated activation in the external channels (see P100 scalp topographies in Fig. 2B). On the other hand, N140 showed a comparable activation area across stimuli (see N140 scalp topographies in Fig. 2B).

The frontal ROI (see ROI-P240 in Fig. 2C) generated the peak around 240ms, it was almost overlapped across stimuli except for SP-650, that showed the highest amplitude, whereas the scalp topographies were very similar in amplitude and site of activation in the frontal area (see Fig. 2C).

Finally, the PS peak showed similar temporal evolution and comparable scalp topographies across stimuli (see Fig. 2D).

\subsection{Modulation of SEPs}

The analysis of P100 amplitude and latency is reported in Fig. 3A and B respectively. The CLH amplitude showed the highest values for the stimuli SP-650 and SP-800 and comparable value for stimuli with SP450 and SP-1050. On the other hand, the ILH amplitude was comparable across stimuli except for SP800. This variability generated no statistical difference between CLH and ILH amplitudes as confirmed also by the scalp distribution across stimuli (see ROI-CLH and ROI-ILH Fig. 2A and 2B, respectively). Regarding the latency, CLH values were comparable across stimuli, whereas ILH values were significantly higher than the CLH ones $[\mathrm{F}(1,3)=145, \mathrm{p}<0.001$, high effect size 1.2].

The N140 amplitude showed comparable CLH values across stimuli whereas the ILH reported a slightly decreasing trend when increasing the roughness (see Fig. 3C). The CLH and ILH amplitudes were also significantly different $[\mathrm{F}(1,3)=10.03, \mathrm{p}<0.01$ medium effect size 0.36$]$. The N140 latency did not 
change across stimuli, whereas the delayed ILH latency values were significantly higher than the CLH ones $[F(1,3)=159, \mathrm{p}<0.001$, high effect size 1.12] (see Fig. 3D).

The frontal P240 SEP showed a nonlinear amplitude trend (Fig. 3E), whereas the latency showed a slightly decreasing trend when increasing the roughness of the stimuli (Fig. 3F).

The PP amplitude was used to evaluate the modulation of the P100 - N140 sequence across stimuli (Fig. 3G). The CLH values showed a decreasing trend when increasing the coarseness of the stimulus, whereas the ILH values changed in a nonlinear way and they were statistically different to the CLH ones $[\mathrm{F}(1,3)$ $=9.23, \mathrm{p}=<0.01$, medium effect size 0.30$]$.

The P100-N140 time lag did not change across stimuli and it was comparable between sides (see Fig. 3H). The P100 - P240 time lag (Fig. 3I) decreased linearly with the coarseness in the CLH, whereas the ILH showed a nonlinear trend but with a significant difference between CLH and ILH $[\mathrm{F}(1,3)=33$, $\mathrm{p}<$ 0.001, high effect size 0.65]. Inversely, the N140-P240 temporal lag reported a distinct decreasing trend across stimuli both in CLH and ILH side, with lower amplitude in the ILH side $[\mathrm{F}(1,3)=40, \mathrm{p}<0.001$, high effect size 0.63$]$.

\section{3 $\theta E R S$ and $\alpha E R D$ characterization}

The time-frequency maps for $\theta \mathrm{ERS}$ and $\alpha \mathrm{ERD}$ have been reduced to "single channel" by averaging the time series of ROI-CLH and ROI-ILH (see Fig. 4A) channels and the results are reported in Fig. 4B. Each stimulus (thus not dependent on its roughness) consistently generated the two sequential bilateral processes described in (Genna et al., 2017): i) $\theta \mathrm{ERS}$, consisting in the increment of the power in theta band with respect to the baseline, starting after the beginning of the stimulation and lasting around 500 ms (see continuous red data in TF maps in Fig. 4B); ii) $\alpha E R D$, which represented a power decrease in the alpha band with respect to the baseline, overlapping for a brief interval to $\theta \mathrm{ERS}$ and lasting throughout the sliding phase before returning to baseline level after 500ms. 
The continuous $\theta \mathrm{ERD}$ and $\alpha \mathrm{ERD}$ above each time-frequency map in Fig. 4B show the overlap in time of the two phenomena, occurring around $250 \mathrm{~ms}$. The continuous $\alpha \mathrm{ERD}$ was comparable across stimuli and sides, whereas the continuous $\theta$ ERD showed lower maximum amplitude in the ipsilateral side for all stimuli. This is also confirmed by the scalp topographies in Fig. 4C. The qERS scalp maps reported some variability in amplitude but the area of activation was comparable across stimuli. The qERD showed comparable amplitude in the CLH and ILH, whereas the area of activation, localized in the somatosensory cortex of both hemispheres, spread out when a stimulus with smaller SP was delivered.

\subsection{Stimulus modulation of $\theta$ ERS and $\alpha$ ERD}

The modulation of $\theta \mathrm{ERS}$ and $\alpha \mathrm{ERD}$ across stimuli is reported in Fig. 5. The CLH max values of the continuous $\theta$ ERS showed a slightly decreasing trend when increasing the SP, whereas the ILH values were comparable across stimuli and were statistically smaller than the CLH ones $[\mathrm{F}(1,3)=14.65, \mathrm{p}<$ 0.001, medium effect size 0.34] Fig. 5A. The latency, i.e., temporal occurrence of the maximum value, was comparable across sides and across stimuli (see Fig. 5B). In case of continuous $\alpha$ ERD, both the maximum value and latency were comparable across stimuli and sides (see Fig. 5C - D).

Finally, the activation of the $\theta \mathrm{ERS}$ scalp maps (Fig. 5E), quantified as average of the channels ROI-CLH and ROI -ILH in Fig.4A, showed comparable values across stimuli but statically smaller values in the ipsilateral side $[\mathrm{F}(1,3)=6.18, \mathrm{p}<0.05$, medium effect size 0.25$]$. On the other hand, the $\alpha \mathrm{ERD}$ was comparable across sides: a slightly linear decreasing trend was reported in the CLH and the same trend was also found in the ILH group. 


\section{Discussion}

In this study we performed a sensory discrimination task during which we stimulated the human fingertip by passively sliding textured surfaces while monitoring the cortical activity by means of EEG. We aimed at i) investigating the neural responses generated by using gratings with fine to medium roughness (i.e., different Spatial Period - Fig. 1C) and ii) identifying the cortical representation of tactile roughness in both contralateral and ipsilateral hemispheres.

\subsection{Brain responses to fine-to-medium tactile stimuli}

In line with the analysis protocol in (Genna et al., 2017) we first verified that the selected stimuli generated a physiological sequence of SEPs. The passive sliding of each grating elicited the following long-latency SEPs consistent across stimuli (see Fig. 2): bilateral P100, bilateral N140, frontal P240 and final PS. The sequence P100-N140 was located in the contralateral and ipsilateral hemispheres as reported in Fig. 2A-B and it is compatible with neural changes in the somatosensory cortex where bilateral generators are present (Hämäläinen et al., 1990). The strong and early activation of the somatosensory area could be dependent on the stimulation modality ( $\mathrm{Ku}$ et al., 2007), whereas the consistency of SEPs across stimuli could be explained by the type of protocol we used (i.e., no-estimation task), that is known to induce activation mainly in the somatosensory area (Kitada et al., 2005).

The P240, similar across stimuli, could represent the prefrontal or Anterior Cingulate Cortex (ACC) activation that suggests engagement of non-specific functions such as spatial attention at one body side (i.e., right finger) (Klimesch, 2012; Ku et al., 2007) or a shift in concentration due to the alternation of the sliding phase and waiting for the next stimulus (Polich, 2007).

The consistency across stimuli confirms the classification of PS as a component located in the sensory functional area encoding the end of the tactile input (Schomer and Da Silva, 2012), regardless of the stimulus roughness (Fig. 2D). 


\subsection{Roughness modulation in the time domain}

The amplitude and latency of SEPs were modulated by the stimulus roughness as reported in Fig. 3. The amplitude of N140 in the CLH was significantly higher than in the ILH, with a slightly linear decreasing trend when increasing the roughness (see Fig. 3C). On the other hand, both P100 and N140 showed very similar CLH latencies across stimuli and significant delay in the ipsilateral side with respect to CLH (see Fig. 3B-D). The tactile roughness seems to modulate amplitude and latency of the SEPs while maintaining the neurophysiological relation between CLH and ILH responses (Garcia-Larrea et al., 1995; Genna et al., 2017).

To determine how the roughness would affect the sequence of these somatosensory potentials we also evaluated the Peak-to-Peak amplitude (PP) and the reciprocal temporal lag of P100 and N140. The PP decreased when increasing the stimulus roughness (Fig. 3G) and it was significantly higher in the CLH with respect to ILH (see Fig. 3G). The temporal lag between P100 and N140, instead, was comparable across stimuli for both the CLH and ILH. These results suggest that the P100 and N140 build the early sequence of the cortical processing of touch, whereas the tactile roughness modulates mainly the PP amplitude in the contralateral side.

The frontal P240 reported similar amplitudes across stimuli except for SP-650, whereas the latency decreased when increasing the roughness (see Fig. 3F): the stimulus roughness linearly modulated the latency of frontal potentials, i.e., smoother surfaces generated delayed potentials. The prefrontal activation could be a result of some level of cognitive-based processing as shown in (Burton et al., 1997) and therefore the observed behavior could be related to the ability of the subject to recognize fine/coarse surfaces: a coarse surface is easier to discriminate and thus might evoke an earlier response (Ballesteros et al., 2009). Previous studies described the involvement of P240 also in different cognitive tasks and experimental settings. For example, (Yamasaki et al., 2000) showed a reduction in amplitude of the N140-P240 pain-related somatosensory evoked potentials during distracting tasks such as calculation 
and memorization. (Wang et al., 2003) investigated the effect of sleep on pain-related SEP and highlighted a significant decrease in N130-P240 amplitude during sleep with respect to vigilance, thus demonstrating the link with cognitive functions and consciousness. Other studies showed that P240 amplitude is also directly involved in recognition tasks, e.g., visual discrimination of emotional and neutral expressions (Gan et al., 2009). Accordingly, the interaction between early (P100 and N140) and late (P240) potentials shown by our data may be linked to the cognitive processing of touch and may highlight an attention-modulated early feature recognition process for tactile surface texture encoding (Reinacher et al., 2009).

In the following analyses we combined the information coming from the somatosensory cortex (P100 and N140 i.e., sensory processing) and the pre-frontal cortex (P240 i.e., recognition processing) by evaluating the temporal lag of P100-P240 and N140-P240 in both CLH and ILH and ipsilateral hemispheres. Both cases showed a linear decreasing of the CLH latency when increasing the roughness (Fig. 3I and 3L). Most interestingly, this trend was maintained also in the ILH side of N140-P240 (Fig. 3L) and it suggests that the stimulus roughness modulates the N140-P240 latency similarly in both hemispheres.

This result might indicate a similar representation of the stimulus roughness both in the contralateral and ipsilateral sides, thus supporting the bilateral representation of touch (Tamè et al., 2016) and the functional involvement of the ipsilateral hemispheres in touch processing (Kitada et al., 2005; Staines et al., 2002). Further studies may also focus on the effect of handedness by replicating the experiment with the non-dominant hand.

\subsection{Roughness modulation in time-frequency domain}

In the time-frequency domain, the two cortical phenomena showed in (Genna et al., 2017) could be robustly identified also across stimuli (see Fig. 4B). The first process was the $\theta \mathrm{ERS}$, i.e., the power 
increase in the theta band occurring in the $[0500] \mathrm{ms}$ interval. The second process was the $\alpha \mathrm{ERD}$, i.e., decrease of the alpha power, which lasted throughout the passive stimulation.

The $\theta$ ERS was clearly detectable with all the stimuli both in the contralateral and in the ipsilateral side albeit with a lower amplitude (Fig. 4B - C). The amplitude of $\alpha$ ERD was instead comparable across both contralateral and ipsilateral hemispheres but showed variability across stimuli (Fig. 4C, right column). The amplitude of continuous $\theta \mathrm{ERS}$ was statistically different between CLH and ILH (Fig. 5A) but similar across stimuli, while continuous $\alpha$ ERD showed comparable amplitude across sides and stimuli (Fig. 5C). The $\theta \mathrm{ERS}$ and $\alpha \mathrm{ERD}$ can be considered as two parallel processes overlapped across time as showed by the continuous data in Fig. 4B. The $\alpha$ ERD is overlapped to the $\theta \mathrm{ERS}$ but with a delayed activation. Most likely this delay is related to feeling adaptation phenomena involving both hemispheres (Genna et al., 2017).

Both $\theta$ ERS and $\alpha$ ERD reached their maximum amplitude at comparable latencies for all the stimuli (Fig. $5 \mathrm{~B}, \mathrm{D})$, suggesting that the $\theta \mathrm{ERS}$ and $\alpha \mathrm{ERD}$ overlap might be a basic cortical mechanism for the processing of touch and stimulation modality (passive sliding of textured surfaces over finger), rather than the tactile roughness modulation.

The qERS average amplitude was comparable across stimuli and was significantly higher for CLH than ILH qERS (see Fig. 5E and scalp topography, Fig. 4C). Conversely, the qERD amplitude exhibited a linear decreasing trend when increasing the tactile roughness without significant differences between CLH and ILH (Fig. 5F and scalp topography, Fig. 4C). The stimulus roughness modulated the alpha band similarly in the ipsilateral and contralateral sides and could be therefore considered a fundamental part of the cortical processing, engaging both hemispheres (Jones et al., 2007; Staines et al., 2002).

The modulation of the $\alpha E R D$ has been shown to reflect the activation of specific neural mechanisms concerning attention, which have a crucial role both in sensory processing (Giabbiconi et al., 2007; Hlushchuk and Hari, 2006) and stimulus modulation (Stancak et al., 2003). A lower engagement of the 
subject in the discrimination task when presented with an easier recognizable surface might explain the linear amplitude reduction of $\alpha \mathrm{ERD}$ with the increase of stimulus roughness that we reported here.

\subsection{Significance for rehabilitation and the development of hand neuroprostheses with sensory feedback.}

The consistent bilateral SEP activation also corroborates recent findings that highlight the importance of the ipsilateral hemisphere for rehabilitation. For instance (Bundy et al., 2017) were able to improve motor recovery in chronic hemiparetic stroke survivors by means of a powered exoskeleton driven by a brain computer interface (BCI), using neural activity from the unaffected (i.e. contralesional) cortical hemisphere. This suggests that the unaffected hemisphere may have great therapeutic potential not only for tactile feedback restoration but also for BCI-driven neurorehabilitation approaches. Our findings are also potentially relevant in the development of prostheses for tactile feedback restoration. Despite the significant functional gain represented by myoelectric prosthetic hands, allowing upper limb amputees to perform several everyday living tasks, prostheses are often rejected by patients, chiefly due to their lack of sensory feedback (Biddiss and Chau, 2007; Engdahl et al., 2015) and cognitive workload required for their use. Depending on the sensory modality, sensory feedback strategies can be classified as nonhomologous (feedback provided via a different sensory modality, e.g., vibro-tactile stimulation) or homologous (the restored sensation matches the original sensation, e.g., invasive electrical stimulation of the sensory fibers), non-somatotopic (e.g, vibro-tactile stimulation of the arm to convey hand tactile feedback) or somatotopic (the restored sensation matches in position the original sensation, e.g., on the phantom limb)(Raspopovic et al., 2014; Zhang et al., 2015). The homologous and somatotopic approach allows immediate and effortless incorporation of the feedback within the sensory motor scheme, without the need of specific training (D'anna et al., 2017). Here we showed that tactile stimuli, even marginally different, result in a specific modulation of SEPs in the time and time-frequency domains. The 
methodology presented here (experimental set up and analysis technique) can thus be used in further studies to test the level of sensory substitution effected by somatotopic and homologous stimulation, i.e. its effectiveness in allowing discrimination of fine textures. To this aim it is possible to replicate the experiment described in (Oddo et al., 2016) using fine-textured gratings to determine, for instance, the level of reorganization, if any, at the cortical level, thus verifying the actual limits of the approach. qERS and qERD measures (especially $\alpha$ ERD) might also serve as a means to reliably measure the cognitive workload in myoelectric prostheses control and human-machine interaction and, accordingly, guide prostheses development (Deeny et al., 2014). Reducing the attentional needs for myoelectric control would make basic activities of daily living, such as dressing, toileting, and ambulation, much easier for amputees, thus greatly increasing the attainable quality of life (Resnik et al., 2012).

\subsection{Conclusions}

Tactile stimuli with fine to medium roughness generated consistent physiological bilateral SEP sequences, which allowed the investigation of the stimuli modulation in both contralateral and ipsilateral hemispheres. We evaluated different aspects in the time and frequency domains and determined that the cortical representation of roughness was modulated similarly in the contralateral and ipsilateral hemispheres in terms of two distinctive features. The first is the temporal lag between N140 and P240. This effect synthesizes both the sensory processing of the somatosensory cortex (N140) and the encoding and cognitive processing of the tactile stimulus (P240). The second is the $\alpha E R D$ average amplitude, comparable in trend and amplitude across hemispheres and related to the attention mechanisms.

On the contrary, the $\alpha \mathrm{ERD}$ and $\theta \mathrm{ERS}$ time overlap was invariant to the tactile roughness and thus may represent a basic cortical mechanism for the processing of touch.

Future works will focus on the combination of psychophysical protocols and EEG to better understand the neural mechanisms behind the cognitive discrimination task. 


\section{Methods and materials}

\subsection{Participants}

10 healthy naïve subjects, (4 females mean population age: 22.4 years) with no deficit of neurological and somatosensory diseases were recruited. The subjects were the same as in (Genna et al., 2017). All experimental procedures were conducted in accordance with the Declaration of Helsinki. Written informed consent for participation in the study was obtained.

\subsection{Tactile experimental setup}

The mechanical tactile stimuli were textured surfaces (see Fig. 1A) fabricated with 3D printing (Project HD 3000, 3D Systems). The spatial coarseness of these stimuli varied from fine to medium coarseness (Weber et al., 2013) and was evaluated by considering the Spatial Period (SP), i.e., the sum of the ridge and groove width (see Fig. 1B). The selected textured stimuli had the following SPs: 450 $\mu \mathrm{m}, 650 \mu \mathrm{m}, 800 \mu \mathrm{m}$ and $1050 \mu \mathrm{m}$ (see Fig. 1C).

A mechatronic platform (Genna et al., 2016; Oddo et al., 2011; Oddo et al., 2016) was used to deliver the mechanical stimulation as passive sliding of a grating under the subject fingertip. The device had 2 Degrees of Freedom (DoFs), which allowed indentation (i.e., vertical motion of the stimulus for the direct contact between stimulus and fingertip) and sliding (i.e., horizontal motion to perform the stimulation). During the mechanical stimulation, a 64ch HD-EEG device (SD LTM Express, Micromed S.p.A, Italy) was used to record the EEG activity (sample rate as $1024 \mathrm{~Hz}$ ). The montage was in accordance with the 5\% 10/20 system and the electrode impedance was kept below $10 \mathrm{k} \Omega$ (Oostenveld and Praamstra, 2001).

\subsection{Stimulation procedure}

Each volunteer sat in a chair and the right arm was positioned on an ergonomic arm support in order to fix the index finger securely to the mechatronic platform by means of a digit holder. The load 
force was set to $400 \mathrm{mN}$, the sliding velocity to $10 \mathrm{~mm} / \mathrm{s}$ and these values were kept constant during the experimental protocol. During the stimulation, the subjects were instructed to focus on the sensory responses generated by the contact stimulus-finger. All subjects were able to complete the task without lamenting any difficulties or fatigue.

The delivery of a tactile stimulus consisted of the following steps: i) indentation of the stimulus (i.e., the finger is in contact with the stimulus) performed smoothly in $4 \mathrm{~s}$; ii) -pause (2s) to avoid artifacts on the EEG signals due to the indentation; iii) start of passive sliding, lasting 2s (i.e., tactile stimulation); iv) pause to isolate post-stimulus potentials (2s); v) removal of the stimulus and start of a new trial. Afferent adaptation was avoided by guaranteeing an inter-trial interval longer than 4s (Leung et al., 2005). The four stimuli were delivered in a random sequence and a large number of repetitions (52) was collected per each stimulus to ensure a robust time-frequency analysis (Graimann et al., 2002).

\subsection{EEG processing}

\subsubsection{Data pre-processing}

EEG data were re-referenced to TP7/TP8, high-pass filtered $(0.5 \mathrm{~Hz}, 94$ th order, Chebyshev type II filter) and a $50 \mathrm{~Hz}$ comb notch filter was applied (Artoni et al., 2017; Menicucci et al., 2014). Channels with artifacts were identified by visual inspection and excluded from the analysis (Artoni et al., 2012a; Sebastiani et al., 2015). Epochs with high-amplitude, high-frequency muscle noise and other irregular artifacts were removed and remaining data were processed via Independent Component Analysis (ICA) filtering to remove non-neural sources (Artoni et al., 2012b; Artoni et al., 2014). EEG trials were then selected from $-2 \mathrm{~s}$ to $4 \mathrm{~s}$ time locked to the onset of the stimulus sliding and subsequently the baseline value (i.e., mean in [-2 0$]$ s) was subtracted from each repetition. No more than $10 \%$ of trials were removed as a result of the preprocessing. Finally, single trial Z-scores were computed by dividing the EEG signals during the stimulation ([2 4]s) by the baseline standard deviation (Delorme and Makeig, 
2004). Z-scores were averaged across subjects to obtain the Grand-Average (GA), i.e., the population mean.

\subsubsection{Identification of SEPs across stimuli}

In our previous publication (Genna et al., 2017), SEPs generated by this stimulation consisted in the bilateral sequence P100-N140, the frontal P240 and the central PS (the final peak occurred around $200 \mathrm{~ms}$ after the end of the stimulation). Moreover, each SEP showed a specific area of activation represented by a significant cluster of electrodes (Genna et al., 2017). Briefly, a channel was assembled to a significant cluster and a time-window qualified as evoked potential, if the interval length of consecutive significant samples was higher than that in the baseline. More in details, for each channel we evaluated whether each time sample had an amplitude statistically different from zero (t-test, $\mathrm{p}$-value $<0.05)$. False positives were reduced by computing the mean duration of the interval of consecutive significant samples in the baseline. This value was used as a threshold to qualify an interval of consecutive significant samples as an evoked potential. For each temporal window, specific for P100, N140, P240 and PS, electrodes that contained a significant interval within that window, formed clusters that identified the specific location of the component on the scalp.

In this study, this procedure has been applied separately for each stimulus and the shared clusters of electrodes were then synthesized in four Regions of Interest (ROIs) (see Fig. 2): 1) contralateral ROI (ROI-CLH, channels: C3, C5, CP3, CP5, P5) 2) ipsilateral ROI (ROI-ILH, channels: C4, C6, CP4, CP6, P6); 3) frontal ROI (ROI-P240, channels: F1, Fz, F2, FC1, FC2, C1, C2, Cz) and 4) central ROI (ROIPS, channels: FC1, Fz, FC2, C1, C2, Cz, CPz). The P100 - N140, P240 and PS were respectively identified by ROIs 1 and 2, 3, 4. The channels of each ROI were averaged to obtain a representative signal allowing the direct comparison across stimuli (named in the following text as roi-signal).

The SEP scalp topographies were computed from the roi-signals by using the temporal windows estimated in (Genna et al., 2017) as follows: (i) maximum in [50 120]ms and [80 160]ms for the CLH 
and ILH P100, respectively; (ii) minimum in [120 140]ms and [180 240]ms for the CLH and ILH N140, respectively; (iii) maximum in [220 260]ms for the P240 and iv) maximum in [2150 2250]ms for the PS.

\subsubsection{Somatosensory Evoked Potentials analysis}

To evaluate the modulation of the brain responses due to the stimulus roughness in the contralateral and ipsilateral hemispheres we first analyzed the amplitude and latency features of SEPs.

The amplitude index was computed as the mean over a $60 \mathrm{~ms}$ temporal window centered at the relevant $\max$ (or min) of the potentials respectively in CLH and ILH ROIs for P100 and N140, frontal ROI for P240. Additionally, we computed the peak-to-peak amplitude of P100-N140 estimated as the sum of the max values of P100 and N140 in both CLH and ILH.

The latency was computed as the temporal occurrence of the max (or min) of the identified SEPs. In particular, we measured the CLH and ILH latencies for both P100 and N140 as well as the latency of $\mathrm{P} 240$.

The temporal lag of the main SEP potentials was also evaluated by computing the latency difference between P100 and N140, P100 and P240, N140 and P240 for both contralateral and ipsilateral hemispheres.

The results for each feature are reported as group value (i.e., mean of the population) and the related standard error. Statistical differences across stimuli and across sides were evaluated via a two-way repeated measures ANOVA ( $1^{\text {st }}$ level - hemisphere, $2^{\text {nd }}$ level - SP, Mathworks Matlab v9.3) and Tuckey’s Honestly Significance Difference Post-hoc test. Effect sizes were calculated with G*Power v3.1.9.2 $\left(1^{\text {st }}\right.$ level - hemisphere, G*Power Statistical test "Means: Difference between two independent means, $\mathrm{n} 1=$ n2"), and rated effect sizes as small, medium, high if respectively in the order of magnitude of 0.10 , 0.25, 0.45 (Faul et al., 2009). 


\subsubsection{Event-related Synchronization/Desynchronization analysis}

Synchronization (ERS) and Desynchronization (ERD) were computed as reported in (Pfurtscheller and Lopes da Silva, 1999) and described briefly here. Each trial was bandpass-filtered $\left(5^{\text {th }}\right.$ order, Butterworth filter) and then squared. The data were smoothed by averaging over trials and over samples. The significant time-frequency maps were then obtained by performing a bootstrap analysis with significance set at 5\% (Graimann et al., 2002). Finally, the Grand-Average was computed by averaging significant time-frequency maps across subjects.

Two frequency bands of interest were selected and we calculated the synchronization/desynchronization activity as follows: 1) $\theta \mathrm{ERS}$, i.e., power increase in theta band ([4 7] Hz); 2) $\alpha \mathrm{ERD}$, i.e., power decrease in alpha band ([8 15] Hz). The same time-frequency maps were computed here per each stimulus.

To summarize the contralateral and ipsilateral contributions of each stimulus, two single-channel timefrequency maps were obtained by averaging the channels of the ROI-CLH (channels: FC5, FC3, FC1, C5, C3, C1, CP5, CP3, P5, P3) and the channels of ROI-ILH (channels: FC6, FC4, FC2, C6, C4, C2, CP6, CP4, P4, P6) (see Fig. 4A) defined in (Genna et al., 2017).

Additionally, the continuous $\theta \mathrm{ERS}$ and $\alpha \mathrm{ERD}$ were extracted from the single-channel maps as the mean in the theta and alpha bands, respectively, in order to monitor the overlapping activity across time.

Finally, the scalp topographies of $\theta \mathrm{ERS}$ and $\alpha \mathrm{ERD}$ were obtained by first averaging the time-frequency maps in the related bands and then by performing the time integral in the interval $\left[\begin{array}{ll}0 & 0.5\end{array}\right] \mathrm{s}$ and $\left[\begin{array}{l}0.252 \\ 2\end{array}\right] \mathrm{s}$ for $\theta \mathrm{ERS}$ and $\alpha \mathrm{ERD}$ respectively.

\subsubsection{Modulation of $\theta E R S$ and $\alpha E R D$ across stimuli}

Both continuous $\theta$ ERS and $\alpha$ ERD were modulated by the different stimuli. In the scalp topography, the channels of the ROI-CLH and ROI-ILH were averaged to quantify the $\theta$ ERS (qERS) and $\alpha \operatorname{ERD}(\mathrm{qERD}$ ) activity and thus compare these values across stimuli and sides. The maximum value over time and its temporal occurrence for $\theta \mathrm{ERS}$ and $\alpha \mathrm{ERD}$ were regarded as features. 
The results are showed as population mean (i.e., Grand-Average) of the computed features and the related standard error. The statistical difference between sides and stimuli were evaluated via a two-way repeated measures ANOVA, (see section 4.4.3).

\section{References}

Allison, T., McCarthy, G., Wood, C.C., 1992. The relationship between human long-latency somatosensory evoked potentials recorded from the cortical surface and from the scalp. Electroencephalography and Clinical Neurophysiology. 84, 301-314.

Arthurs, O.J., et al., 2000. Linear coupling between functional magnetic resonance imaging and evoked potential amplitude in human somatosensory cortex. Neuroscience. 101, 803-6.

Artoni, F., et al., 2012a. REMOV: EEG artifacts removal methods during Lokomat lower-limb rehabilitation. In: 2012 IEEE RAS \& EMBS international conference on Biomedical Robotics and Biomechatronics Vol., ed.^eds. IEEE, pp. 992-997.

Artoni, F., et al., 2012b. ErpICASSO: a tool for reliability estimates of independent components in EEG event-related analysis. In: 2012 34th Annual International Conference of the IEEE Engineering in Medicine and Biology Society (EMBC). Vol., ed.^eds. IEEE, pp. 368-371.

Artoni, F., et al., 2014. RELICA: a method for estimating the reliability of independent components. NeuroImage. 103, 391-400.

Artoni, F., et al., 2017. Unidirectional brain to muscle connectivity reveals motor cortex control of leg muscles during stereotyped walking. Neuroimage. 159, 403-416.

Backes, W.H., et al., 2000. Somatosensory cortex responses to median nerve stimulation: fMRI effects of current amplitude and selective attention. Clinical neurophysiology : official journal of the International Federation of Clinical Neurophysiology. 111, 1738-44.

Ballesteros, S., Reales, J.M., 2004. Visual and Haptic Discrimination of Symmetry in Unfamiliar Displays Extended in the $<\mathrm{i}>\mathrm{z}</ \mathrm{i}>$-Axis. Perception. 33, 315-327.

Ballesteros, S., et al., 2009. ERP evidence of tactile texture processing: Effects of roughness and movement. In: World Haptics 2009 - Third Joint EuroHaptics conference and Symposium on Haptic Interfaces for Virtual Environment and Teleoperator Systems. Vol., ed.^eds. IEEE, pp. 166-171.

Bergmann Tiest, W.M., Kappers, A.M.L., 2007. Haptic and visual perception of roughness. Acta Psychologica. 124, 177-189.

Biddiss, E.A., Chau, T.T., 2007. Upper limb prosthesis use and abandonment: a survey of the last 25 years. Prosthetics and orthotics international. 31, 236-257.

Bodegård, A., et al., 2000. Somatosensory areas in man activated by moving stimuli: cytoarchitectonic mapping and PET. Neuroreport.

Bundy, D.T., et al., 2017. Contralesional brain-computer interface control of a powered exoskeleton for motor recovery in chronic stroke survivors. Stroke. 48, 1908-1915.

Burton, H., et al., 1997. Multiple foci in parietal and frontal cortex activated by rubbing embossed grating patterns across fingerpads: a positron emission tomography study in humans. Cereb Cortex. 7, 317.

Coghill, R.C., et al., 1994. Distributed processing of pain and vibration by the human brain. The Journal of neuroscience : the official journal of the Society for Neuroscience. 14, 4095-108. 
Connor, C.E., et al., 1990. Tactile roughness: neural codes that account for psychophysical magnitude estimates. J Neurosci. 10, 3823-36.

D'anna, E., et al., 2017. A somatotopic bidirectional hand prosthesis with transcutaneous electrical nerve stimulation based sensory feedback. Scientific reports. 7, 10930.

Deeny, S., et al., 2014. A simple ERP method for quantitative analysis of cognitive workload in myoelectric prosthesis control and human-machine interaction. PloS one. 9, e112091.

Delorme, A., Makeig, S., 2004. EEGLAB: an open source toolbox for analysis of single-trial EEG dynamics including independent component analysis. Journal of neuroscience methods. 134, 921.

Engdahl, S.M., et al., 2015. Surveying the interest of individuals with upper limb loss in novel prosthetic control techniques. Journal of neuroengineering and rehabilitation. 12, 53.

Faul, F., et al., 2009. Statistical power analyses using G* Power 3.1: Tests for correlation and regression analyses. Behavior research methods. 41, 1149-1160.

Francis, S., et al., 2000. fMRI of the responses to vibratory stimulation of digit tips. Neuroimage.

Gan, T., et al., 2009. Emotional influences on time perception: evidence from event-related potentials. Neuroreport. 20, 839-843.

Garcia-Larrea, L., Lukaszewicz, A.-C., Mauguiere, F., 1995. Somatosensory responses during selective spatial attention: The N120-to-N140 trasition. Psychophysiology. 32, 526-537.

Genna, C., et al., 2016. Long-latency components of somatosensory evoked potentials during passive tactile perception of gratings. in 2016 38th Annual Int. Conf. of the IEEE Engineering in Medicine and Biology Society (EMBC). accepted.

Genna, C., et al., 2017. Spatiotemporal Dynamics of the Cortical Responses Induced by a Prolonged Tactile Stimulation of the Human Fingertips. Brain Topography. 1-13.

Giabbiconi, C.-M., et al., 2007. Sustained spatial attention to vibration is mediated in primary somatosensory cortex. NeuroImage. 35, 255-262.

Graimann, B., et al., 2002. Visualization of significant ERD/ERS patterns in multichannel EEG and ECoG data. Clin Neurophysiol. 113, 43-7.

Hämäläinen, H., et al., 1990. Human somatosensory evoked potentials to mechanical pulses and vibration: contributions of SI and SII somatosensory cortices to P50 and P100 components. Electroencephalography and clinical neurophysiology. 75, 13-21.

Harada, T., et al., 2004. Asymmetrical neural substrates of tactile discrimination in humans: a functional magnetic resonance imaging study. Journal of.

Hlushchuk, Y., Hari, R., 2006. Transient Suppression of Ipsilateral Primary Somatosensory Cortex during Tactile Finger Stimulation. Journal of Neuroscience. 26, 5819-5824.

Iguchi, Y., et al., 2002. Selective attention regulates spatial and intensity information processing in the human primary somatosensory cortex. Neuroreport. 13, 2335-9.

Johansson, R.S., Flanagan, J.R., 2009. Coding and use of tactile signals from the fingertips in object manipulation tasks. Nature Reviews Neuroscience. 10, 345-59.

Jones, S.R., et al., 2007. Neural Correlates of Tactile Detection: A Combined Magnetoencephalography and Biophysically Based Computational Modeling Study. Journal of Neuroscience. 27.

Kaczmarek, K.A., et al., 1991. Electrotactile and vibrotactile displays for sensory substitution systems. IEEE Transactions on Biomedical Engineering. 38, 1-16.

Kitada, R., et al., 2005. Tactile estimation of the roughness of gratings yields a graded response in the human brain: an fMRI study. NeuroImage. 25, 90-100.

Klatzky, R.L., Lederman, S.J., Metzger, V.A., 1985. Identifying objects by touch: An “expert system”. Perception \& Psychophysics. 37, 299-302. 
Klimesch, W., 2012. alpha-band oscillations, attention, and controlled access to stored information. Trends Cogn Sci. 16, 606-17.

$\mathrm{Ku}, \mathrm{Y}$., et al., 2007. Prefrontal cortex and somatosensory cortex in tactile crossmodal association: an independent component analysis of ERP recordings. PLoS One. 2, e771.

Leung, Y.Y., et al., 2005. Time-course of vibratory adaptation and recovery in cutaneous mechanoreceptive afferents. Journal of neurophysiology. 94, 3037-45.

Menicucci, D., et al., 2014. Brain responses to emotional stimuli during breath holding and hypoxia: an approach based on the independent component analysis. Brain Topogr. 27, 771-85.

Munoz, F., et al., 2014. An electrophysiological study of haptic roughness: effects of levels of texture and stimulus uncertainty in the P300. Brain Res. 1562, 59-68.

Nelson, A.J., et al., 2004. Activation in SI and SII; the influence of vibrotactile amplitude during passive and task-relevant stimulation. Cognitive Brain Research. 19, 174-184.

Oddo, C.M., et al., 2011. A mechatronic platform for human touch studies. Mechatronics. 21, 604-613.

Oddo, C.M., et al., 2016. Intraneural stimulation elicits discrimination of textural features by artificial fingertip in intact and amputee humans. Elife. 5, e09148.

Oostenveld, R., Praamstra, P., 2001. The five percent electrode system for high-resolution EEG and ERP measurements. Clin Neurophysiol. 112, 713-9.

Pfurtscheller, G., Lopes da Silva, F.H., 1999. Event-related EEG/MEG synchronization and desynchronization: basic principles. Clinical neurophysiology. 110, 1842-57.

Polich, J., 2007. Updating P300: an integrative theory of P3a and P3b. Clin Neurophysiol. 118, 2128-48.

Raspopovic, S., et al., 2014. Restoring natural sensory feedback in real-time bidirectional hand prostheses. Science translational medicine. 6, 222ra19-222ra19.

Reinacher, M., et al., 2009. Oscillatory brain states interact with late cognitive components of the somatosensory evoked potential. Journal of Neuroscience Methods. 183, 49-56.

Resnik, L., et al., 2012. Advanced upper limb prosthetic devices: implications for upper limb prosthetic rehabilitation. Archives of physical medicine and rehabilitation. 93, 710-717.

Roland, P., O'Sullivan, B., 1998. Shape and roughness activate different somatosensory areas in the human brain. Proceedings of the.

Salenius, S., et al., 1997. Modulation of human cortical rolandic rhythms during natural sensorimotor tasks. NeuroImage. 5, 221-8.

Schomer, D.L., Da Silva, F.L., 2012. Niedermeyer's electroencephalography: basic principles, clinical applications, and related fields, Vol., Lippincott Williams \& Wilkins.

Sebastiani, L., et al., 2015. Inefficient stimulus processing at encoding affects formation of high-order general representation: A study on cross-modal word-stem completion task. Brain research. 1622, 386-396.

Staines, W.R., et al., 2002. Task-Relevant Modulation of Contralateral and Ipsilateral Primary Somatosensory Cortex and the Role of a Prefrontal-Cortical Sensory Gating System. NeuroImage. 15, 190-199.

Stancak, A., et al., 2003. Desynchronization of cortical rhythms following cutaneous stimulation: effects of stimulus repetition and intensity, and of the size of corpus callosum. Clin Neurophysiol. 114, 1936-47.

Stoeckel, M., et al., 2003. A fronto-parietal circuit for tactile object discrimination:: an event-related fMRI study. Neuroimage.

Tamè, L., et al., 2016. Bilateral representations of touch in the primary somatosensory cortex. Cogn Neuropsychol. 1-19.

Taylor, M.M., Lederman, S.J., 1975. Tactile roughness of grooved surfaces: A model and the effect of friction. Perception \& Psychophysics. 17, 23-36. 
Torquati, K., et al., 2002. Comparison between SI and SII responses as a function of stimulus intensity. Neuroreport. 13, 813-9.

Wang, X., et al., 2003. Effects of sleep on pain-related somatosensory evoked potentials in humans. Neuroscience research. 45, 53-57.

Weber, A.I., et al., 2013. Spatial and temporal codes mediate the tactile perception of natural textures. Proceedings of the National Academy of Sciences. 110, 17107-12.

Yamasaki, H., et al., 2000. Effects of distraction on pain-related somatosensory evoked magnetic fields and potentials following painful electrical stimulation. Cognitive Brain Research. 9, 165-175.

Yoshioka, T., et al., 2001. Neural coding mechanisms underlying perceived roughness of finely textured surfaces. J Neurosci. 21, 6905-16.

Zhang, D., et al., 2015. Somatotopical feedback versus non-somatotopical feedback for phantom digit sensation on amputees using electrotactile stimulation. Journal of neuroengineering and rehabilitation. 12, 44 .

\section{Figure legend}

\section{Figure 1: Tactile stimuli and roughness scale.}

A) Tactile stimuli (gratings), i.e., mechanical stimuli with alternated ridges and grooves on the topside.

B) The roughness was defined by the Spatial Period (SP) i.e., the sum of the Ridge Width (RW) and

Groove Width (GW). In this study, the ridge width and Ridge Height $(\mathrm{RH})$ were maintained constant and only the GW was varied to modulate the spatial coarseness. C) Lower SP produced a finer stimulus whereas higher SP and GW values indicated coarser stimuli. In the scale from fine to medium roughness, four tactile stimuli were selected with the following SP: $450 \mu \mathrm{m}, 650 \mu \mathrm{m}, 800 \mu \mathrm{m}$ and $1050 \mu \mathrm{m}$.

\section{Figure 2: Stimulus-dependent characterization of SEPs.}

A) Significant electrodes of the contralateral cluster (ROI-CLH) and temporal evolution of SEPs (i.e., average of the channels in ROI-CLH) of each stimulus. The reported scalp distributions represent the $\max$ in $[0.050 .1]$ s for the P100 and min in $[0.08$ 0.15]s for N140 across stimuli. Both P100 and N140 are generated systematically across stimuli reporting some variability in amplitude and latency. B) 
Significant electrodes of the ipsilateral cluster (ROI-ILH) and temporal evolution of ipsilateral SEPs, computed as average of the channels of ROI-ILH. The scalp distributions were evaluated as max in [0.1 0.17]s for P100 and as $\min$ in $[0.18$ 0.24]s for N140. The topography of P100 shows variability, whereas the ipsilateral N140 is elicited systematically across stimuli. C) Significant electrodes of the frontal cluster (ROI - P240) and temporal evolution, computed as average of the electrodes of ROI-P240. The P240 scalp topography was evaluated as max in [0.19 0.28]s and it shows similar site of activation across stimuli. D) Significant electrodes of the central cluster (ROI-PS) and temporal evolution, computed as average of the electrodes of ROI-PS. The PS peak was identified as max in [2.14 2.26]s and it is comparable across stimuli.

\section{Figure 3: Roughness modulation across SEP features.}

A) - B) P100 amplitude and latency distribution across stimuli. C) - D) N140 amplitude and latency across stimuli. E) - F) P240 amplitude and latency showing a slightly decreasing trend from the fine to coarse stimulus $(\Delta(450-1050)=32 \mathrm{~ms})$. G) Peak-to-peak of P100-N140 distribution across stimuli. H) Time lag between P100 and N140, it maintains similar values across stimuli and across sides. I) Time lag P100-P240. It shows a decreasing trend $(\Delta(450-1050)=36 \mathrm{~ms})$, very similar for 800 and 1050 for CLH whereas the ILH shows a nonlinear trend. L) Time lag N140-P240. It shows a linear decreasing trend when moving from fine to rough stimuli both in CLH and ILH sides. Significance levels $*=p<0.05$; $* *=\mathrm{p}<0.01 ; * * *=\mathrm{p}<0.001$.

\section{Figure 4: Bilateral representation of $\theta \mathrm{ERS} / \alpha \mathrm{ERD}$ across stimuli.}

A) Time frequency (TF) maps of the selected channels in ROI-CLH and ROI-ILH, averaged to provide a single map of contralateral and ipsilateral responses of each stimulus. B) The left and right columns 
show the contralateral and ipsilateral TF maps respectively, whereas each row represents the tactile stimulus that generated the responses. Above each TF maps, the continuous $\theta \mathrm{ERS}$ (red line) and $\alpha \mathrm{ERD}$ (blue line) are also reported (equal axis [-10 60]\%). Each stimulus generated two consecutive processes: increase of theta power between $\left[\begin{array}{ll}0 & 0.5\end{array}\right] \mathrm{s}$ and decrease the alpha power between $\left[\begin{array}{ll}0.25 & 2\end{array}\right] \mathrm{s}$. The overlapping of the two phenomena are clearly visible in the continuous signals. The $\alpha$ ERD is comparable across sides, whereas the $\theta$ ERS shows the highest peak in the contralateral side for all the stimuli. C) $\theta$ ERS and $\alpha$ ERD were quantified as time integral in the temporal windows $\left[\begin{array}{ll}0 & 0.5\end{array}\right] \mathrm{s}$ and $\left[\begin{array}{ll}0 & 2\end{array}\right] \mathrm{s}$, respectively. The scalp distributions are slightly variable across stimuli but with comparable area of activations.

\section{Figure 5: Roughness modulation across time-frequency features.}

A) - B) Max values of continuous $\theta$ ERS and their temporal occurrence for each stimulus. The CLH max values are significantly higher than ILH ( $\mathrm{p}<.00001$, two-way ANOVA). C) - D) Max values of continuous $\alpha$ ERD and their temporal occurrence across stimuli. E) $-F$ ) Integrated $\theta \mathrm{ERS}$ and $\alpha \mathrm{ERD}$, quantified as average of the channels in the selected CLH and ILH ROI. $\theta \mathrm{ERS}$ amplitude is very similar across stimuli and significantly higher than ipsilateral $(\mathrm{p}=.0047$, two-way ANOVA). $\alpha$ ERD shows a decreasing trend when increasing the stimulus roughness both in the contralateral and ipsilateral hemispheres. Significance levels $*=p<0.05 ; * *=p<0.01 ; * * *=p<0.001$.

\section{Contributions}

CG designed the study, developed the experimental set up, performed the experiments, analyzed the data, discussed the results and wrote the paper. CMO co-designed the study, developed the tactile stimulation platform, supervised its experimentation and discussed the results. CF performed the experiments. CC 
provided neurophysiological background and discussed the results. SM co-supervised the experiments, co-designed the study, discussed the results and wrote the paper. FA designed the study, supervised the experiments, analyzed the data, discussed the results and wrote the paper. All authors reviewed and approved the final manuscript.

\section{Acknowledgments}

This work was supported by the EU Grant FET 611687 NEBIAS Project (NEurocontrolled BIdirectional Artificial upper limb and hand prosthesiS) and by the Ronda project, Robotica indossabile personalizzata per la riabilitazione motoria dell'arto superiore in pazienti neurologici. Regione Toscana, Bando FAS

Salute 2014 PAR FAS 2007-2013. Dr. Artoni's contributions were supported by the European Union's Horizon 2020 research and innovation programme under Marie Skłodowska Curie grant agreement No. 750947 (project BIREHAB).

\section{Appendix}

EEG: $\quad$ Electroencephalogram; electroencephalography;

SEP: $\quad$ Somatosensory evoked potential

P100, N140, P240, PS: $\quad$ SEPs respectively at 100, 140, $240 \mathrm{~ms}$ after the start of sliding and after s timulation ends (PS)

ROI Region of interest, i.e. group of channels clustered in a particular region of the berain

ROI-P100, ROI N140,

ROI-P240, ROI-PS

Region of interest associated to the P100, N140, P240, PS SEPs

CLH, ILH

Contralateral hemisphere, Ipsilateral hemisphere

ROI-CLH, ROI-ILH Contralateral and Ipsilateral ROIs 
$\alpha$ ERD, $\theta$ ERS

qERD, qERS

SP

GA
Event related desynchronization and synchronization respectively in the $\alpha$ and $\theta$ bands

maximum value over time of $\alpha \mathrm{ERD}$ and $\theta \mathrm{ERS}$ respectively, averaged across the channels of ROI-CLH or ROI-ILH

Spatial period of the grating stimulus, sum of Ridge Width (RW) and Groove Width (GW)

Grand average over trials 

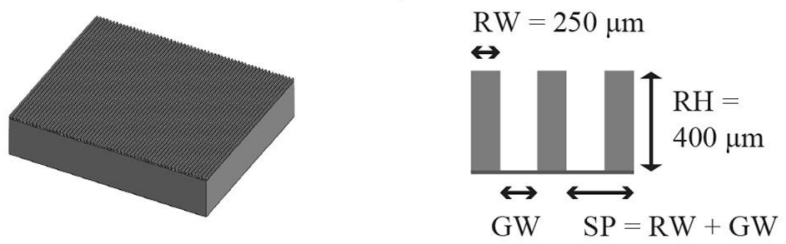

GW $(\mu \mathrm{m})$

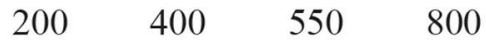

fine

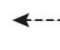

\section{0 \\ 650 \\ 800

1050 

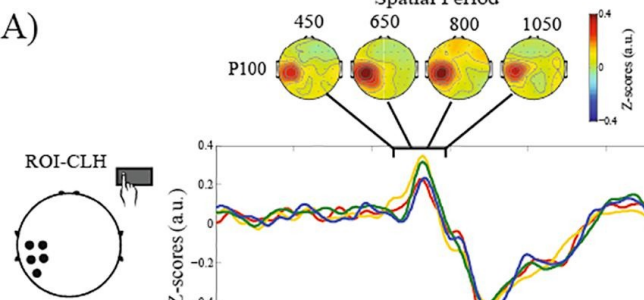

B)
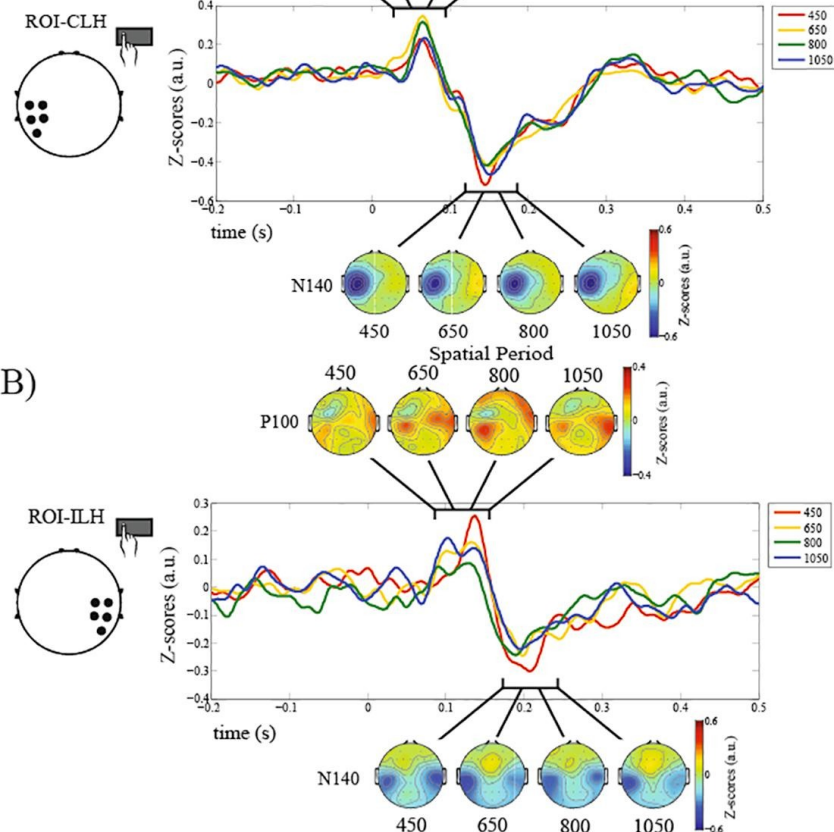

C)

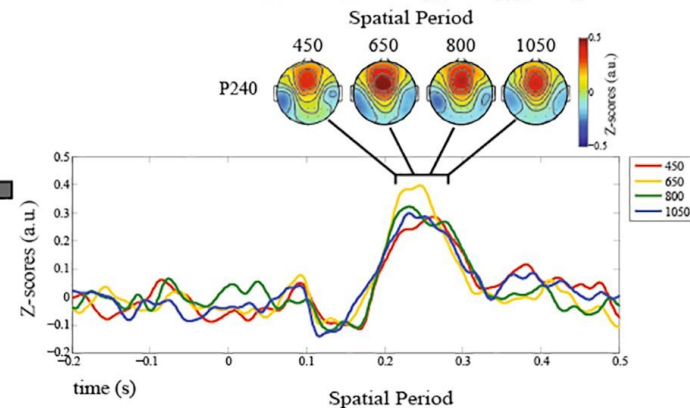

D)

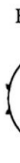

ROI-P240

:a:

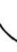

.
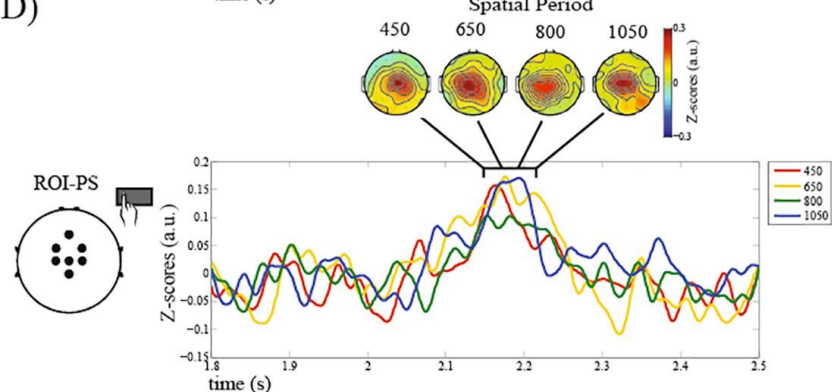
fine $\longleftarrow 450 \square 650 \square 800 \square 1050 \longrightarrow$ coarse

A)

B)

$* * *$
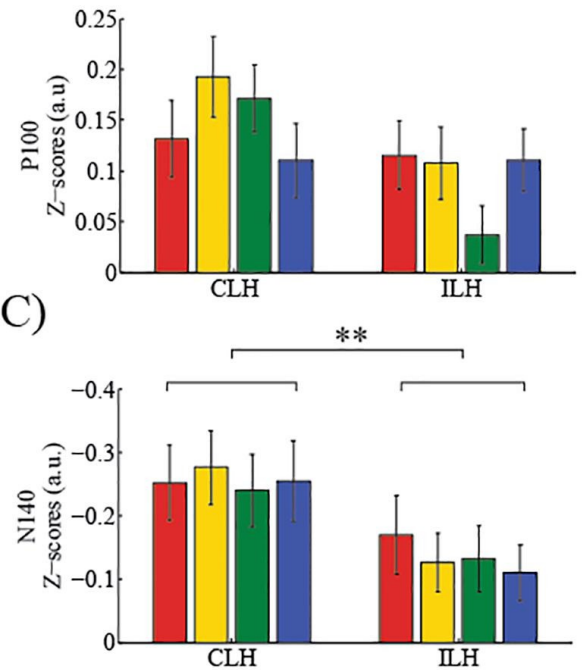

D)

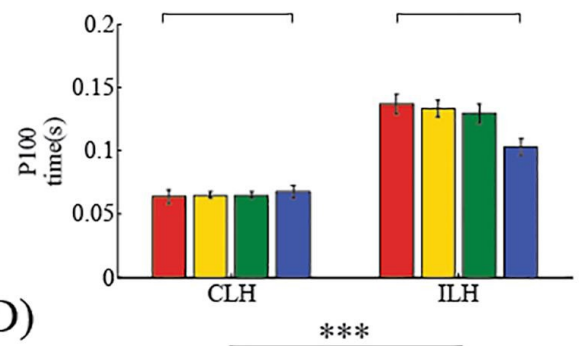

E)

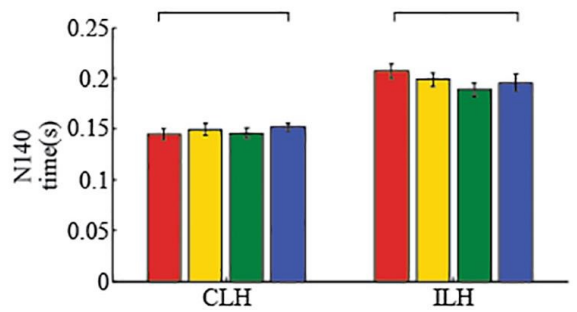

F)
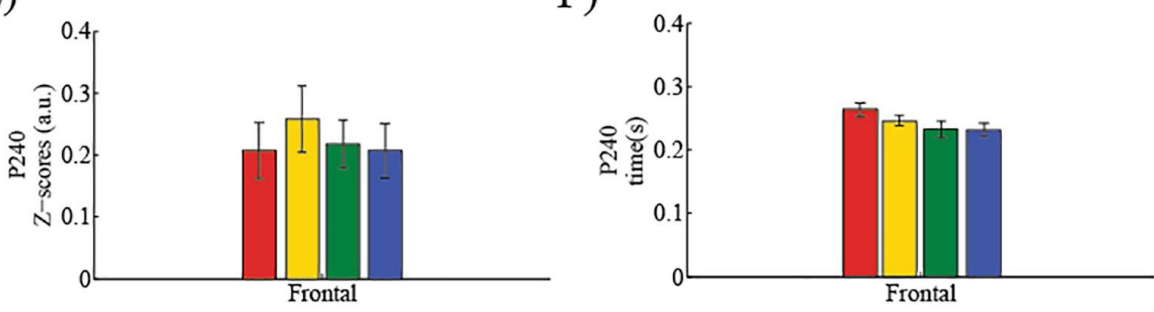

$\mathrm{G}$
I)

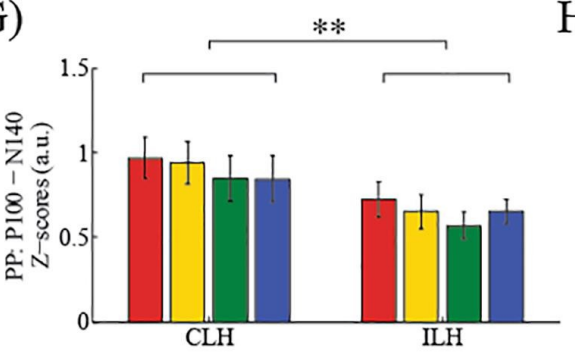

***

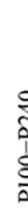

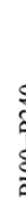

早

0.25

0.2

0.15

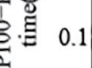
0.05
H)

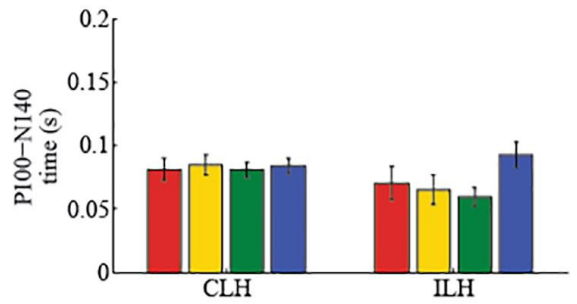

L)

***

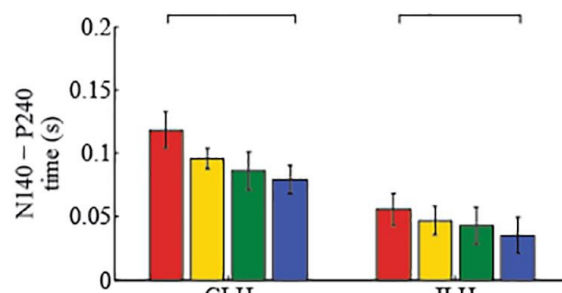




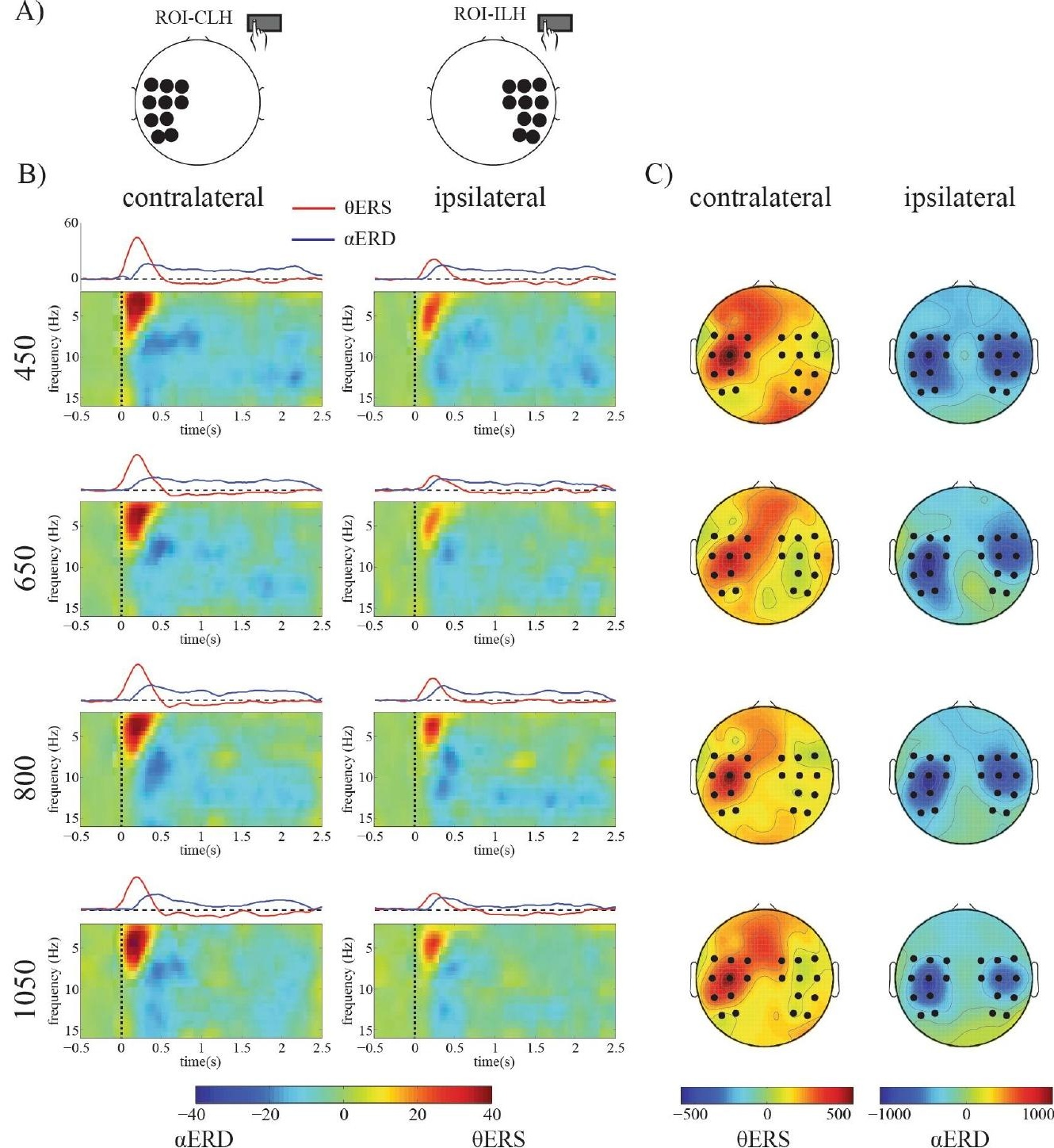


